\title{
Repräsentationen, Diskurse und Machtfragen: Postkoloniale Theorieansätze in der Friedens- und Konfliktforschung
}

Der Beitrag geht der Frage nach, inwiefern postkoloniale Theorie in der Friedensund Konfliktforschung bereits berücksichtigt wird und welche Potenziale diese theoretische Perspektive bietet. Im ersten Schritt erfolgt eine Bestandsaufnahme bestehender Analysen im Bereich der Friedens- und Konfliktforschung, die sich auf postkoloniale Theorie beziehen. Im zweiten Schritt wird diskutiert, welchen spezifischen Beitrag postkoloniale Ansätze für die Forschung über Gewaltkonflikte und Friedensprozesse leisten können. Darauf aufbauend werden konkrete Ansatzpunkte für zukünftige Analysen benannt. Im dritten Schritt wird diskutiert, inwiefern die Gefahr besteht, dass eine stärkere Berücksichtigung durch die breite wissenschaftliche und politische Debatte dem kritischen Anspruch postkolonialer Studien nicht gerecht wird. Gestützt auf die Erfahrung der feministischen Ansätze in der Friedensund Konfliktforschung wird argumentiert, dass diese Gefahr durchaus existiert. Der Beitrag schließt mit der Forderung, postkoloniale Theorie einschließlich ihres macht- und wissenschaftskritischen Fundaments in den friedenswissenschaftlichen Debatten ebenso wie in der Hochschullehre stärker zu verankern.

Schlagworte: Postkolonialismus, Feminismus, Friedens- und Konfliktforschung, Macht, Wissenschaftskritik

\section{Einleitung}

Postkoloniale Theorie hat in den Sozialwissenschaften auch im deutschsprachigen Raum einige Prominenz erlangt. ${ }^{1}$ Davon zeugen in jüngster Zeit erschienene Sammelbände (etwa Conrad/Randeria 2002; Reuter/Villa 2010 a; Rodríguez et al. 2010), Schwerpunktausgaben unterschiedlicher Fachzeitschriften ${ }^{2}$ und neu geschaffene,

1 Für hilfreiche Anmerkungen zu diesem Beitrag danke ich Sarah Clasen, Lars Schmitt, Birgitta Wodke sowie der Redaktion, den Gutachter_innen und den Herausgeber_innen der ZeFKo.

2 Etwa Femina Politica 2/2009: »Feministische postkoloniale Theorie: Gender und (De-)Kolonialisierungsprozesse«, Prokla 158/2010: »Postkoloniale Studien als kritische Sozialwissenschaft« sowie PERIPHERIE 120/2010: »Postkoloniale Perspektiven auf 〉Entwicklung««. 
teilweise bereits institutionell verankerte Forschungsschwerpunkte. ${ }^{3}$ In der Friedens- und Konfliktforschung schlägt sich dieser Trend bislang kaum nieder, zumindest nicht in Form entsprechender Publikationen. Vice versa nehmen postkoloniale Studien selten explizit Bezug auf die Friedens- und Konfliktforschung. Trotzdem wächst in Letzterer - auch im deutschsprachigen Raum - das Interesse an postkolonialen Theorieansätzen. Nicht zuletzt fordern Studierende die Berücksichtigung dieser Ansätze in den Zusammenhängen des universitären Lehrens und Lernens. ${ }^{4}$

Ziel dieses Beitrags ist es, auszuloten, wie postkoloniale Theorieansätze in der Friedens- und Konfliktforschung Anwendung finden (können). Ich gehe dafür in drei Schritten vor: Zunächst werden die Grundzüge postkolonialer Theorieansätze skizziert und anhand exemplarischer Arbeiten dargestellt, inwiefern sie bereits in der Friedens- und Konfliktforschung aufgegriffen werden. Dabei lassen sich Arbeiten, die das Attribut »postkolonial« nicht im Sinne postkolonialer Studien, sondern als räumlich-historische Kategorie verwenden, von solchen unterscheiden, denen es darum geht, die Potenziale postkolonialer Theorien für die Analyse von Krieg oder Sicherheit sichtbar zu machen. Diese Arbeiten sind vor allem im Bereich der Internationalen Beziehungen (IB) zu finden. Eine dritte Gruppe stellen Beiträge dar, die spezifische Fragestellungen aus dem Bereich der Friedens- und Konfliktforschung unter Zuhilfenahme von Bestandteilen postkolonialer Theorie, oft kombiniert mit anderen, beispielsweise feministischen theoretischen Zugängen, bearbeiten. Im zweiten Schritt wird diskutiert, was postkoloniale Ansätze zur Friedens- und Konfliktforschung beitragen können, um daran anschließend Ansatzpunkte für entsprechende postkoloniale Analysen zu skizzieren. Darauf folgend frage ich im dritten Schritt nach den Chancen und Risiken der Berücksichtigung postkolonialer Ansätze in der breiten wissenschaftlichen und politischen Debatte. Hierzu greife ich auf die Erfahrung feministischer Theorien als einer weiteren kritischen sozialwissenschaftlichen Perspektive zurück, die vergleichsweise bereits stärker in der Friedens- und Konfliktforschung wahrgenommen wird. Dies bietet sich an, da feministische und postkoloniale Ansätze eine miteinander verschränkte Wissenschafts- und Herrschaftskritik als konstitutiven Theoriebestandteil gemeinsam haben. Die feministische Erfahrung zeigt, dass kritische Perspektiven erfolgreich darin sein können, in der breiten Debatte gehört zu werden. Gleichzeitig wird deutlich, dass eine solche

3 An der Goethe-Universität Frankfurt wurde ein eigenes Research Center for Postcolonial Studies eingerichtet und eine Juniorprofessur für Politikwissenschaft mit dem Schwerpunkt Gender/Postkoloniale Studien geschaffen (www.frcps.uni-frankfurt.de; letzter Zugriff: 16.02.2014).

4 Siehe etwa die von Studierenden über den Verein Commit e. V. im Wintersemester 2009/10 organisierte Veranstaltungsreihe $»$ Wissensmacht - Machtwissen« an der Humboldt-Universität Berlin. 
Berücksichtigung dem politisch und theoretisch emanzipativen Kern feministischer Perspektiven nicht immer gerecht wird.

Der Einbezug postkolonialer Perspektiven in die Friedens- und Konfliktforschung könnte in ähnlich ambivalenter Weise verlaufen. Eine Forschung, die auf das Element der Wissenschafts- und Herrschaftskritik verzichtet, wäre allerdings mit »postkolonial« im theoretischen Sinne unzutreffend bezeichnet. Dabei bieten postkoloniale Ansätze gerade für die Friedens- und Konfliktforschung im deutschsprachigen Raum nicht nur die Chance, dass »blinde Flecken« und mögliche Schieflagen sichtbar werden, indem als selbstverständlich geltende Ausgangspunkte in der Forschung hinterfragt werden, sondern auch den Anschluss an eine breitere sozialwissenschaftliche Debatte, die international und vor allem im englischen Sprachraum bereits weiter fortgeschritten ist als hierzulande. Der Beitrag schließt mit der Forderung, postkoloniale Theorie einschließlich ihres macht- und wissenschaftskritischen Kerns für die wissenschaftlichen Debatten innerhalb der Friedens- und Konfliktforschung fruchtbar zu machen und sie auch in der Hochschullehre im deutschsprachigen Raum stärker zu verankern.

\section{Postkoloniale Perspektiven auf Konflikt und Frieden}

Postkoloniale Theorie lässt sich schwerlich als ein in sich geschlossenes Theoriegebäude verstehen, sondern eher als ein Spektrum theoretisch-analytischer Perspektiven auffassen. Gemeinsam ist ihnen, dass sie "postkolonial « nicht als historisch-räumliche Kategorie verstehen: Die Vorsilbe »post« bezeichnet nicht die Trennung eines »Davor« (oder »Während «) und »Danach« kolonialer Herrschaft. Den Bezugspunkt der Theoriebildung und empirischen Analyse stellt vielmehr die Wirkmächtigkeit des Kolonialismus bis in die Gegenwart dar - in Politik, Gesellschaft und Wissenschaft in den ehemaligen Kolonien ebenso wie in den ehemaligen Kolonialmächten (Aikins/Bendix 2010; Randeria/Eckert 2009). Die dichotome Konstruktion von »kolonialer« und »postkolonialer« Epoche sowie der Fokus auf die ehemaligen Kolonien bei Ausblenden der Kolonialmächte würden den konstitutiv-produktiven Wechselbeziehungen der kolonialen Begegnung nicht gerecht (Hall 2002; vgl. auch Ashcroft et al. 1995; Castro Varela/Dhawan 2005; Kapoor 2008). »Postkolonial« ist

»als eine politisch motivierte Analysekategorie zu verstehen, die einerseits die nachhaltige Prägung der globalen Situation durch Kolonialismus, Dekolonialisierung und neokolonialistische Tendenzen aufzeigt - und damit nicht nur die aktuelle Wirkmächtigkeit eines unabgeschlossenen Kolonialdiskurses 
konstatiert, sondern auch ein chronologisches Geschichtsverständnis kritisiert« (Reuter/Villa 2010 b: 7).

Dabei stellen Wissenschafts- und Herrschaftskritik konstitutive erkenntnistheoretische Elemente postkolonialer Theorie dar: Es gilt, die Verschränkung von Wissen und Macht offenzulegen. Postkolonial angeleitete Arbeiten dekonstruieren das Bild eines »aufgeklärten« und »zivilisierten« Westens, das sich in als selbstverständlich hingenommenen Dichotomien wie Tradition und Moderne, Barbarei und Zivilisation, Natur und Kultur eingeschrieben hat (Hall 1992).

Der Fokus auf die Analyse von Repräsentation und Diskursen, auf Sprache und Symbole erklärt sich durch die Ursprünge postkolonialer Theorie in den Literaturund Kulturwissenschaften. Mit Verweis auf diesen disziplinären Ursprung sei »den postkolonialen Studien in der Vergangenheit die Relevanz für sozialwissenschaftliche Forschung weitgehend abgesprochen worden «(Franzki/Aikins 2010: 9). Jedoch erscheint die (selbst-)kritische postkoloniale Analyse für die Sozialwissenschaften bereits insofern notwendig, als schon die Auffächerung des sozialwissenschaftlichen disziplinären Kanons die Konstruktion kolonial verankerter Dualismen widerspiegelt: Soziologie und Politikwissenschaft richten den Blick überwiegend auf die »modernen« Gesellschaften des Westens, während der »Rest« (Hall 1992) den Regionalwissenschaften und der Ethnologie überlassen bleibt (Ziai 2011: 25).

Kritisiert wurden postkoloniale Ansätze insbesondere aus materialistischer Perspektive dafür, die Bedeutung des Kapitalismus für die weltgeschichtliche Entwicklung zu vernachlässigen (Ahmad 1995; Dirlik 1994). Postkoloniale Theorie sieht sich mit dem Vorwurf konfrontiert, sich in theoretischen Sphären des discursive und cultural turn zu verlieren - auf Kosten des kritisch-emanzipatorischen Anspruchs und der Anschlussfähigkeit an die politische Praxis. Arif Dirlik (1994) argumentiert, dass die postkoloniale Theorie nur deshalb so prominent in den westlichen Wissenschaften werden konnte, weil sie die materielle Basis globaler Abhängigkeits- und Herrschaftsverhältnisse zugunsten von Diskurs und Repräsentation theoretisch zurückgestellt habe. Mit dieser analytisch-politischen Entschärfung gehe die fehlende Thematisierung der Auswirkungen neoliberaler Globalisierung auf die Nord-Süd-Abhängigkeitsverhältnisse einher. Diese sei eine wesentliche Voraussetzung für die Akzeptanz postkolonialer Studien im westlichen Wissenschaftsbetrieb gewesen.

Im Bereich der Friedens- und Konfliktforschung hat sich das wachsende Interesse an der postkolonialen Theorie bislang kaum in der empirischen Forschung niedergeschlagen. Verglichen mit Themen wie Migration (etwa Ha 2004; Rodríguez 2010; 
Rytkonen 2006) und Entwicklung (etwa Eriksson Baaz 1995; Kapoor 2008; Nandy 2002) befassen sich erst wenige Arbeiten aus postkolonialer Perspektive mit Fragen von Konflikt, Sicherheit und Frieden. Während für andere wissenschaftliche Felder bereits allgemeine Beiträge zu ihren Schnittstellen mit postkolonialen Studien vorliegen (etwa Abrahamsen 2003 für die Afrika- und Sylvester 1999 für die Entwicklungsforschung), steht eine solche Diskussion für die Friedens- und Konfliktforschung noch aus. Die Zusammenfassung dessen, was in diesem Forschungsfeld an postkolonial ausgerichteten Beiträgen vorliegt, fällt nicht allzu umfangreich aus. Dabei können drei Typen unterschieden werden: Erstens wird in der Mehrheit der Arbeiten, die das Adjektiv »postkolonial « im Titel tragen, der Begriff im Sinne einer räumlich-historischen Kategorie verwandt (etwa Blanton et al. 2001; Henderson/ Singer 2000; Mkandawire 2002). Einen Bezug zu postkolonialen theoretischen Perspektiven stellen diese Beiträge nicht her.

Zweitens sind Beiträge zu nennen, die in ihrer Auseinandersetzung mit Krieg oder Sicherheit explizit auf postkoloniale Theorien rekurrieren. Auffällig ist dabei, dass solche Arbeiten vor allem im Bereich der IB (Agathangelou/Ling 2004) und der Sicherheitsforschung vorliegen. $\mathrm{Zu}$ nennen sind etwa die Ausgabe der Zeitschrift Millennium (1/2007) mit dem Schwerpunkt »Edward W. Said and International Relations« und der Einführungsbeitrag von Siba Grovogui (2007). Tarak Barkawi und Mark Laffey (2006) kritisieren die Sicherheitsforschung für das ihr immanente eurozentrische Geschichtsverständnis, das die wechselseitige Bedingtheit der europäischen und nicht-europäischen Welt ausblende und deshalb kein adäquates Verständnis für bewaffneten Widerstand entwickeln könne. Jörg Meyer (2008) stellt aus ähnlicher Perspektive die Legitimität militärischer Interventionen »im Namen des Friedens « infrage. Diese gründe sich auf einem Selbstbild des Westens als »Friedensbringer« für die »Anderen«, die nicht willens oder in der Lage seien, selbst Frieden zu schaffen.

Drittens schließlich lassen sich Arbeiten finden, die ausgehend oder unter Zuhilfenahme von postkolonialen Theorieansätzen Fragestellungen mit Bezug auf Konflikt, Sicherheit und Frieden untersuchen. Zu nennen sind hier etwa Beiträge, die sich kritisch mit »Terrorismus« befassen. Gefragt wird nach der Bedeutung des Dualismus von »westlich« vs. »nicht-westlich« in Diskursen über Terrorismus und für seine Erscheinungsformen in der »realen Welt« (Boehmer/Morton 2009: 2). Claudia Brunner $(2010$; 2012) analysiert aus wissenschaftssoziologischer Perspektive ausgehend von postkolonial-feministischen Theorieansätzen die Wissensbestände der englischsprachigen Terrorismusforschung. Weiterhin sind der »war on terror « und seine Legitimierung über einen Diskurs der (westlichen) »Moderne« Gegenstand postkolonialer Analysen (Mamdani 2004). Einige kritische, insbeson- 
dere von den Arbeiten Michel Foucaults (2003) und Judith Butlers (2004) inspirierte Studien analysieren den Diskurs um den »war on terror « ohne explizite Referenz auf postkoloniale Theorie (etwa Neal 2008). Im Mittelpunkt stehen die Frage, wie über die Diskursfigur »war on terror« Subjekte und Subjektivitäten konstituiert werden (beispielsweise der »islamische Terrorist «), sowie die Wirkmächtigkeit dieser Diskursfigur zur Begründung und Legitimierung von Politiken. Diese Arbeiten zeigen, wie der »war-on-terror«-Diskurs innerhalb der Gesellschaften widergespiegelt wird, und lösen damit die künstliche Trennung von Außen- und Innenpolitik auf. Infolge rücken Politikfelder ins Zentrum der Friedens- und Konfliktforschung, die zuvor eher ihre Peripherie markierten, etwa Migrations- und Flüchtlingspolitik sowie innere Sicherheit (beispielsweise die Einschränkung bürgerlicher Rechte mit Verweis auf Sicherheit und Terrorismusbekämpfung). Postkolonial angeleitete empirische Analysen liegen darüber hinaus zu medialen und wissenschaftlichen Legitimationen militärischer Intervention vor, insbesondere in Bezug auf die jüngsten Kriege in Afghanistan und im Irak (Klaus/Kassel 2008; Nachtigall 2012) sowie auf den Kosovo-Krieg (Bewernitz 2010).

In dem skizzierten dritten Bereich zeigt sich eine erhebliche Schnittmenge feministischer und postkolonial angeleiteter Forschungen: Hier sind zahlreiche Arbeiten zu finden, die sich auf postkolonial-feministische Theorie beziehen und deren Gegenstände Konflikt, Frieden und Sicherheit sind. Sie weisen in der empirischen Forschung oft eine (theoretisch begründete) Präferenz für bestimmte methodische Vorgehensweisen und Themen auf. Typischerweise greifen sie auf sprachbasierte (insbesondere diskursanalytische) Verfahren zurück und dekonstruieren dominante Diskurse in der politischen Praxis (Nachtigall/Bewernitz 2011) oder Wissenschaft (Brunner 2010).

\section{Was können postkoloniale Ansätze in der Friedens- und Konfliktforschung beitragen?}

Postkoloniale Theorieansätze können in der Friedens- und Konfliktforschung prinzipiell das Gleiche leisten wie in anderen Forschungsfeldern auch. Eine ihrer besonderen Stärken liegt in der Analyse, wie die in der Peripherie verorteten »Anderen « (wobei die Peripherie nicht gleichbedeutend mit den Ländern des Globalen Südens ist) im Zentrum repräsentiert werden und wie die Produktion vermeintlich »objektiven« Wissens über die »Anderen« zu dieser spezifischen Repräsentation beiträgt. Zwar muss sich postkoloniale Theorie generell dem Vorwurf stellen, auf der Ebene der Kritik an der Wissensproduktion zu verharren, obgleich Veränderungen der materiellen Gegebenheiten wie der Institutionen internationaler Politik 
und der global-kapitalistischen Wirtschaftsordnung notwendig wären (Ahmad 1995; Dirlik 1994). Ausgehend von der Grundannahme der Verschränkung von Wissen, Macht und der Ausübung von Herrschaft ist jedoch evident, dass die Produktion von (wissenschaftlichem) Wissen eine zentrale Bedeutung für die gesellschaftliche und politische Praxis hat - zumal in Forschungsfeldern wie der Friedensund Konfliktforschung, für die der Bezug von Wissenschaft und Praxis sowie eine Anwendungsorientierung prägend sind. Aus dieser Sicht können postkoloniale Ansätze die Friedens- und Konfliktforschung um die Kritik an Macht-Wissen-Komplexen bereichern und somit zur Reflexion einer möglichen eigenen, damit verbundenen Komplizenschaft anregen. Dabei gilt es etwa aufzudecken, inwiefern wissenschaftliches Wissen über den »Orient «bzw. die »Anderen« intendiert oder nichtintendiert zur diskursiven Legitimierung von Gewalt (etwa militärischer Intervention) beitragen kann.

Damit soll keinesfalls unterstellt werden, dass unser wissenschaftliches Wissen über Konflikt und Frieden per se dazu beiträgt, Herrschaft zu stabilisieren oder Gewalt zu legitimieren. Ich behaupte auch nicht, dass keine Reflexion über die praxeologischen Implikationen und den politischen Gebrauch unseres friedenswissenschaftlichen Wissens stattfinden würde. Postkoloniale Theorie kann jedoch in zweifacher Weise einen spezifischen Beitrag zu dieser Reflexion leisten: Sie kann zum einen ein begrifflich-konzeptionelles Instrumentarium bereitstellen (vgl. dazu Ashcroft et al. 2000; Smith 1999), das der kritischen Diskussion innerhalb der Friedensund Konfliktforschung über ihre möglichen politischen Verstrickungen ein bisher fehlendes theoretisch-methodisches Fundament anbietet. So lassen sich ausgehend von den vorhandenen Wissensbeständen postkolonialer Studien etwa systematisch orientalistische Diskursfiguren identifizieren. Zum anderen führt uns die Auseinandersetzung mit postkolonialer Theorie zur erkenntnistheoretischen Reflexion über Forschungsfragen und -ziele. Denn zentral für postkoloniale Studien ist der Anspruch, nicht (nur) Wissen über die »Anderen« zu produzieren, sondern dieses stets und notwendigerweise mit der Frage zu verbinden, mit welchen Konstruktionen des »Eigenen« dieses Wissen einhergeht.

Eng damit verbunden ist die Forderung, sich der eigenen (Sprecher_innen-)Position bewusst zu werden. Hierbei geht es nicht nur darum, zu diskutieren, wie unsere Forschungsergebnisse in Diskursen und Politiken verwandt werden, wobei diese Verwendung häufig außerhalb unseres Einflussbereichs stattfindet. Im Vordergrund steht auch nicht die - wichtige und in der Friedens- und Konfliktforschung mittlerweile breit diskutierte - methodische Frage, welchen Einfluss der kulturelle Kontext einer Feldforschung bzw. einer Interviewsituation, soziale Rollen, Erwartungen und Machtasymmetrien zwischen Forscher_innen und Forschungssubjekten auf durch 
Interviews produzierte Daten ausüben und welche forschungsethischen Probleme damit verbunden sind (vgl. dazu Buckley-Zistel 2007; Nordstrom 1995; Wood 2006). Die Problematisierung der Sprecher_innen-Position verknüpft Dipesh Chakrabartys (2000) Forderung, Europa zu provinzialisieren, d. h. nicht (länger) als vermeintlichen Ursprung der Geschichte (und der Theorie, etwa der politischen Philosophie) zu setzen, damit, Subjekte und ihre Positionen in der Forschung selbst sichtbar zu machen. Es geht um die Frage, wer in der Forschung zu wem und zu welchem Zweck spricht und wer dabei wen zu repräsentieren in Anspruch nimmt (Steyerl/Rodríguez 2003; Yildiz 1999). Damit verbunden ist stets die Frage, wer vermeintlich nicht spricht oder sprechen kann - Gayatri Spivaks (1988) berühmte Frage, ob die Subalterne sprechen könne. Zudem geht es darum zu dekonstruieren, dass nicht-westliches Wissen häufig als »lokales Wissen « gefasst wird, während westlich-wissenschaftliches Wissen vermeintlich abstrakt, kontextunabhängig und womöglich universell gültig sei (Hacker 2012; Smith 1999).

Wo bestehen nun konkrete Ansatzpunkte für postkoloniale Analysen von bewaffneten Konflikten und Friedensprozessen? Viel Material bieten beispielsweise Berichte und Studien über bewaffnete Konflikte in Afrika. Unter den Schlagwörtern »ethnischer« und »neuer« Kriege wird in Texten und Bildern implizit, manchmal auch explizit, ein »barbarisches« Schwarzes und/oder muslimisches »Anderes" konstruiert, das Gewalt aus Emotion oder Tradition ausübt und zum Frieden nicht willens oder fähig ist. So glichen »im größten Teil Afrikas [...] die kriegführenden Einheiten Volksstämmen - tatsächlich handelt es sich um Stämme oder um das, was nach der zersetzenden Wirkung der Zivilisation noch übrig geblieben ist « (van Creveld 1998: 288, Hervorh. im Orig.), wie Martin van Creveld schreibt. Und »im bettelarmen Äthiopien spielen sich ähnliche Auseinandersetzungen zwischen christlichen Ethnien und islamischen Stämmen ab. Anderswo stehen sich Stammesgruppen gegenüber, die sich nach Sprache und Aussehen unterscheiden « (Hartmann 2001: 204). Aus postkolonialer Perspektive wird die Konstruktion von »ethnischen « Kriegen und »Stammeskriegen « hingegen als koloniale Erfindung enttarnt. So verweist Jean-Loup Amselle (1999: 40) darauf, dass Bauernaufstände und andere Bewegungen gegen die Kolonialisierung als »ethnische« Kriege oder »Stammeskriege« markiert worden seien, um auf diese Weise antikoloniale Widerstände diskursiv unsichtbar zu machen.

Über solche Diskursfiguren wird das »westliche« Selbst mit Attributen wie »modern« oder »zivilisiert« ausgestattet. Ähnliche Dualismen lassen sich beispielsweise in der These von der »Barbarisierung « oder »Brutalisierung « der »neuen Kriege« identifizieren, die geographisch in Afrika und dem Vorderen Orient verortet und den europäischen »Staatenkriegen« gegenüber gestellt werden (etwa Kaplan 1994; 
Münkler 2002; zur Kritik vgl. Kalyvas 2001; Marchal 2000; Richards 1996; Ziai/ Schaffar 2011). Während es in Europa zu einer »Disziplinierung« des Militärs gekommen sei, habe »in Regionen, in denen nomadisierende Hirten- und Jägervölker das Kriegswesen bestimmten [...], die Gewaltanwendung, die ganz selbstverständlich zum Alltagsleben der Nomadenvölker gehörte, [...] fast nahtlos in Krieg übergehen « (Münkler 2002: 117) können.

An postkolonialer Theorie orientierte Studien haben gezeigt, wie Freiwillige und Hauptamtliche in Entwicklungszusammenarbeit und Humanitärer Hilfe eigene Identitäten eines »humanitär« und »partnerschaftlich« helfenden Selbst konstruieren (Eriksson Baaz 1995; Kontzi 2011). Daran können Analysen zu Post-KonfliktKontexten anschließen und den Blick auf die Selbst-Bilder der militärischen und nicht-militärischen Beteiligten von Interventionen der Konfliktbearbeitung und Friedensförderung richten.

Schwerpunkt postkolonialer Beiträge zu Konflikt, Sicherheit und Frieden stellen bislang Interventionen des »Westens« im Orient bzw. im Globalen Süden dar. Viel und noch nicht ausgeschöpftes Potenzial bietet die postkoloniale Theorie darüber hinaus dahingehend, theoretisch zentrale Begriffe kritisch auf ihre Provenienz in europäischen und eurozentristischen Theorietraditionen hin zu überprüfen. Dabei ließen sich für die Friedens- und Konfliktforschung grundlegende und vermeintlich selbstverständliche Konzepte, wie die Leitkategorie »staatlich« vs. »nichtstaatlich«, als in einem westlich-kolonialen Verständnis der (Staaten-)Welt verankert entlarven. Das »Modell der ethnisch verwurzelten Nationalkultur als in sich geschlossener Einheit« (Kerner 2010: 246) ist in der gegenwärtigen Forschung über gewaltsame Konflikte noch immer gängig. Shalini Randeria (1999) setzt dem das Konzept der »verwobenen Moderne « entgegen, das sich gegen die vorherrschende modernisierungstheoretische Vorstellung wendet, der »Westen « stelle die (moderne) Norm gesellschaftlicher Entwicklung dar und der »Rest« repräsentiere dessen (traditionale) Vergangenheit und bewege sich im Sinne einer »nachholenden Entwicklung « auf die »moderne« Norm zu. Aufzudecken, dass und wie diese Idee sowohl friedenswissenschaftlich als auch in der friedenspolitischen Praxis verankert ist, könnte ein wesentlicher Beitrag an postkolonialer Theorie orientierter Friedens- und Konfliktforschung sein.

\section{Mainstreaming Postcolonialism?}

Postkoloniale Theorie kann also die Friedens- und Konfliktforschung insofern bereichern, als sie einen veränderten Blick auf oftmals unhinterfragte Begriffe, Dualismen und Diskursfiguren anbietet. Aus postkolonialer Perspektive stellen sich 
Forschungsfragen, die »blinde Flecken« in unserem Wissen über Konflikt- und Friedensprozesse auszuleuchten vermögen. Eine stärkere Berücksichtigung postkolonialer Theorie in den wissenschaftlichen Debatten und Forschungen über bewaffnete Konflikte, ihre Transformation, Bearbeitung und Aufarbeitung ist für die Friedens- und Konfliktforschung ebenso wünschenswert wie für die postkolonialen Studien: Für die Friedens- und Konfliktforschung, weil sie das Spektrum ihrer Forschungsfragen, -perspektiven und -gegenstände erweitern kann, und für die postkolonialen Studien, weil sie ihren macht- und wissenschaftskritischen Blick auf ein Forschungsfeld ausweiten können, mit dem bislang noch wenige Berührungspunkte bestehen. Doch ein Mainstreaming (wissenschafts-)kritischer Perspektiven geht auch mit dem Risiko einher, dass nur einzelne Aspekte aufgegriffen werden und insbesondere die macht- und selbstkritischen Fragen de facto außen vor bleiben.

Mit Mainstreaming ist in diesem Zusammenhang die Berücksichtigung von Theorieansätzen, die sich explizit kritisch gegenüber bestehenden (wissenschafts-)politischen Verhältnissen und den in einem Forschungsfeld vorherrschenden Thesen, methodischen Herangehensweisen und theoretischen Zugängen positionieren, durch den vorherrschenden Diskurs in eben diesem Feld gemeint. Der Begriff ist im Zusammenhang mit »Gender Mainstreaming « entstanden, einer politischen Strategie mit dem Ziel, in der Planung, Umsetzung und Evaluierung von politischen und administrativen Vorhaben die jeweiligen Auswirkungen auf Frauen und Männer sowie Geschlechtergleichheit zu erfassen. Kritisiert wird »Gender Mainstreaming « unter anderem dafür, dass es bestehende Macht- und Herrschaftsverhältnisse nicht infrage stelle, sondern sie reproduziere, indem der Genderbegriff in politische Programme und Projekte sowie Verwaltungshandeln eingebunden wird, ohne die ihnen zugrunde liegenden Strukturen, Machtverhältnisse und Normen grundsätzlich verändern zu wollen (vgl. Kerner 2007: 2-3). Wie ein solcher Prozess der selektiven Berücksichtigung bezogen auf wissenschaftliche Debatten verlaufen kann, wird im Folgenden anhand der feministischen Ansätze in der Friedens- und Konfliktforschung verdeutlicht.

Der Vergleich mit feministischen Zugängen bietet sich insofern an, als postkoloniale und feministische Theorieansätze grundlegende (erkenntnis-)theoretische Gemeinsamkeiten aufweisen, sich hinsichtlich ihrer Berücksichtigung in der Friedens- und Konfliktforschung aber (noch) unterscheiden. Gemeinsam ist beiden Perspektiven, dass sie »Nischen « jenseits der vorherrschenden wissenschaftlichen Debatten und Zugänge darstellen. Für beide Perspektiven ist die Kritik am erkenntnistheoretischen Fundament gegenwärtig dominierender wissenschaftlicher Positionen wichtig und beide wollen die systematischen Leerstellen in der gesellschaftswissenschaftlichen Forschung aufzeigen, die aus deren andro- bzw. eurozentrischer 
Perspektive resultieren. Nicht zufällig greift eine wachsende Zahl an Arbeiten auf feministische und postkoloniale Ansätze zugleich zurück (etwa Castro Varela/Dhawan 2009; Yegenoglu 1998). Während die Kategorie Geschlecht in der Friedensund Konfliktforschung gegenwärtig weitgehend anerkannt wird, steht ein ähnlicher Integrationsprozess für die postkolonialen Theorieansätze hingegen noch aus.

Die Berücksichtigung neuer theoretischer Perspektiven in der Friedens- und Konfliktforschung erfolgt in vielen Fällen vergleichsweise zögerlich. Dies ist einerseits überraschend, verfügt sie als ein multidisziplinäres Forschungsfeld ${ }^{5}$ doch über das Potenzial, frühzeitig theoretische Entwicklungen unterschiedlicher Fachdisziplinen aufzugreifen. Andererseits ist die Friedens- und Konfliktforschung nach wie vor dem dominanten Einfluss der IB im akademischen sowie der Außen- und Sicherheitspolitik im politisch-praktischen Bereich ausgesetzt - die beide kritisch-theoretischen Überlegungen oft eher skeptisch gegenüberstehen. Auch feministische Perspektiven hatten es anfänglich schwer, in der politischen und akademischen Diskussion über Konflikt und Frieden Gehör zu finden - um einiges schwerer als beispielsweise in der Entwicklungs- oder Sozialpolitik, denen als soft-politics-Bereiche weniger politisches Gewicht zugewiesen wird.

Anfang der 1990er Jahre setzte sich schließlich vor dem Hintergrund des Endes der Blockkonfrontation die Einsicht durch, dass sich Krieg und Frieden nicht ausschließlich vor der Folie konventioneller Ansätze der IB erklären lassen. In theoretischer Hinsicht hat zudem die Prominenz liberaler sozialkonstruktivistischer Ansätze in den IB dazu beigetragen, dass Gender dort und ebenso in der Friedens- und Konfliktforschung zunehmend beachtet wurde (Locher/Prügl 2001). Tatsächlich wird in der Friedens- und Konfliktforschung vor allem ein sozialkonstruktivistischer Genderansatz wahrgenommen, für den die Operationalisierbarkeit des Genderkonzeptes für die empirische Forschung eine wichtige Rolle spielt. In vielen gegenwärtigen Arbeiten, die empirisch mit einer »analytischen Kategorie Geschlecht« operieren, spielen die Analyse und Kritik von Macht- und Herrschaftsverhältnissen eher eine nachgeordnete Rolle - anders als in den frühen Arbeiten feministischer Friedens- und Konfliktforschung im deutschsprachigen Raum (etwa Batscheider 1993). Es besteht kein Zweifel daran, dass die Genderforschung feministisch bleibt, solange es »ihr um das Aufzeigen von Ungleichheit, Ungerechtigkeit und Machtbeziehungen und stets auch um die soziale Veränderung gesellschaftlicher wie politischer Verhältnisse« (Wisotzki 2011: 265) geht. Dies trifft allerdings nicht auf

5 »Multidisziplinär« meint hier lediglich die Summe verschiedener Fachdisziplinen. Interdisziplinarität im Sinne der gemeinsamen Arbeit mehrerer Forscher_innen aus unterschiedlichen Fächern findet in der Friedensforschung zumindest punktuell statt, Transdisziplinarität, verstanden als die Auflösung disziplinärer Grenzen, hingegen seltener. 
alle wissenschaftlichen Arbeiten und politischen Programme zu, die mit dem Etikett »Gender« aufwarten.

Es lässt sich darüber streiten, inwiefern Gender tatsächlich in der Friedens- und Konfliktforschung angekommen ist. Keinesfalls wird die Kategorie Geschlecht bereits selbstverständlich in den meisten empirischen Analysen systematisch berücksichtigt. Zumindest aber erscheinen in vielen Fachzeitschriften inzwischen regelmäßig Beiträge aus der genderbezogenen Forschung (etwa Brunner 2012; Bussmann 2012; Clasen/Zwingel 2009) und viele dieser Zeitschriften haben in den letzten Jahren Heftschwerpunkte aus dem Bereich »Gender und Konflikt « vorgelegt. ${ }^{6}$ Einführungs- und Überblicksbände integrieren Geschlechterperspektiven zumindest mittels einzelner Beiträge (etwa Harders 2005; Sturm 2006; Wisotzki 2005; 2011). Dies ist ein Erfolg und den jahrelangen Bemühungen feministischer Wissenschaftler_innen geschuldet, welche für die Berücksichtigung von Geschlechterperspektiven in der Friedens- und Konfliktforschung ebenso wie in anderen Forschungsfeldern stets gegen Widerstände ankämpfen mussten. Allerdings beschränkt sich die Anerkennung überwiegend auf einen theoretisch und politisch moderaten Genderansatz. Bedeutende Teile der umfangreichen (erkenntnis-)theoretischen Debatten - etwa die innerhalb der feministischen Theorie zentrale Auseinandersetzung mit der Materialität des Körpers (Alaimo/Hekman 2007) - werden in der Friedensund Konfliktforschung nach wie vor kaum wahrgenommen. Dass Feminismus und Gender mehr sind als »add women and stir«, gehört zwar mittlerweile (fast) zu den Wissensbeständen aller sozialwissenschaftlichen Fachdisziplinen. Dennoch beschränkt sich die Einbeziehung von Gender in die empirische Forschung teils auf die Verwendung von Geschlecht als einer (binären) Variablen ohne die Berücksichtigung des kritischen erkenntnistheoretischen Fundaments feministischer Ansätze.

Postkoloniale Ansätze werden bislang in der Friedens- und Konfliktforschung noch weniger wahrgenommen als Geschlechterperspektiven. Angesichts der gegenwärtigen Prominenz postkolonialer Theorie in den Sozialwissenschaften insgesamt ist zu erwarten, dass auch die Friedens- und Konfliktforschung diese künftig stärker berücksichtigen wird. Dass dies ähnlich ambivalent verlaufen könnte wie im Fall der feministischen Ansätze, darauf deuten die Prozesse der Etablierung postkolonialer Zugänge in den Sozialwissenschaften generell hin, die innerhalb der postkolonialen Studien bereits kritisch diskutiert werden. Vor dem Hintergrund, dass postkoloniale Theorie vor allem in den Metropolen des Westens produziert

6 Etwa die Ausgabe 1/2010 der Zeitschrift Sicherheit + Frieden mit dem Schwerpunkt »Gender und Sicherheit «. 
wird, sei wenig überraschend, dass sie sich »von einer scharfen kritischen Theorie [...] zu einer $\gg$ Karrierebeförderungsmaschine (Bahri 1995: 71) gewandelt « (Castro Varela/Dhawan 2010: 305) habe. Schon jetzt büße die postkoloniale Theorie viel von ihrem normativ-politischen Gehalt und emanzipativ-transformativen Anspruch ein, wenn sie Eingang in breite sozialwissenschaftliche und politische Debatten findet. So sei zu beobachten, »dass der Begriff >postkoloniak häufig als Euphemismus für bedrohlichere Konzepte wie >Imperialismus und `Neokolonialismus` zum Einsatz kommt« (Castro Varela/Dhawan 2010: 305).

Die Forderung nach der stärkeren Berücksichtigung kritisch-theoretischer Perspektiven erweist sich angesichts des Risikos der »Gefangennahme « als ambivalent. Mainstreaming kann, wie die feministische Erfahrung zeigt, mit dem Verlust der politisch-theoretischen Substanz einhergehen - und zudem die Forderung nach der expliziten Förderung dieser Perspektiven im Wissenschaftsbetrieb erschweren. Diese Ambivalenz lässt sich kaum auflösen. Fest steht, dass eine postkoloniale Perspektive mehr ist als die Beobachtung, dass die meisten gegenwärtigen bewaffneten Konflikte in den ehemaligen Kolonien stattfinden. Aber eine postkoloniale Perspektive auf Konflikt, Frieden und Sicherheit beschränkt sich auch nicht auf die Analyse, wie der Kolonialismus in den dekolonialisierten Gesellschaften fortwirkt und in welcher Weise dies für gegenwärtige soziale, politische und militärische Konflikte von Bedeutung ist. Zentrales Element postkolonialer Theorie ist neben der Dekonstruktion eurozentrischer Grundbegriffe eine kritische Analyse, wie die (kriegführenden, friedensbedürftigen) »Anderen« im wissenschaftlichen Diskurs repräsentiert werden - und wie Wissenschaft damit zum konstitutiven Bestandteil sich selbst stabilisierender globaler Machtstrukturen wird. Um die Potenziale postkolonialer Theorien für die Friedens- und Konfliktforschung vollständig nutzbar zu machen, ist der Bezug auf ihr herrschafts- und wissenschaftskritisches Fundament unverzichtbar.

\section{Schlussbemerkung}

In diesem Beitrag ging es darum, inwiefern postkoloniale Theorieansätze in der Friedens- und Konfliktforschung bereits einbezogen werden und welche Potenziale sie für zukünftige Forschungen über Gewaltkonflikte und Friedensprozesse bieten. Ich habe argumentiert, dass postkoloniale Theorie die Friedens- und Konfliktforschung bereichern kann und sollte, dabei jedoch auch die Gefahr besteht, dass der kritisch-theoretische Anspruch, welcher die postkolonialen Studien auszeichnet, nur in abgeschwächter Weise übernommen wird. Dieses Argument ist auf die Erfahrung 
der feministischen Ansätze gestützt, die in der Friedens- und Konfliktforschung bereits vergleichsweise stärker berücksichtigt werden.

»Würde heute noch jemand offen die Auffassung vertreten, die Kategorie Geschlecht sei der Friedens- und Konfliktforschung >wesensfremd<, so wäre das politisch nicht mehr korrekt « (Birckenbach 2005: 81). Diese auf den ersten Blick gute Nachricht erweist sich aus der Nähe betrachtet als durchaus ambivalent - schließlich ist Feminismus »mehr als >political correctness « (Locher 1996). Mit der Bezugnahme auf eine vermeintlich machtneutrale »analytische Kategorie Geschlecht» bleiben manche Analysen aus feministischer Sicht verkürzt. »Mainstreaming Gender « und die Bewahrung des herrschaftskritischen Moments sind nicht immer problemlos zu vereinen. Bezeichnend ist, dass Geschlechterperspektiven immer noch sekundär sind, wenn es um die Verteilung von Ressourcen im Wissenschaftsbetrieb geht. Bis heute sind sie institutionell in der deutschsprachigen Friedens- und Konfliktforschung nicht verankert und werden kaum gezielt finanziell gefördert (Wisotzki 2011: 265). Wenn Gender vermeintlich in den Fachdisziplinen integriert ist, lässt sich die Forderung nach der institutionellen Verankerung feministischer Theorien in den Hochschulen in Form eigener Lehrstühle und Forschungsschwerpunkte sowie in den Curricula leicht als überflüssig darstellen. So gibt es gegenwärtig in der deutschen Forschungslandschaft keine Bestrebungen, einen eigenen Lehrstuhl für feministische Friedens- und Konfliktforschung zu etablieren oder ein eigenes Forschungszentrum gründen zu wollen, wie es Anfang der 1990er Jahre versucht wurde (Birckenbach 2005: 78; Wisotzki 2011: 247). Nach wie vor sind feministische oder Geschlechter-Perspektiven in keiner Denomination für einen friedenswissenschaftlichen Lehrstuhl an einer deutschen Hochschule explizit enthalten (Clasen/Zwingel 2009). Die Verankerung von Gender in den Curricula (etwa durch die Einführung verpflichtender als mit einem »Gender-Schwerpunkt« gekennzeichneter Lehrveranstaltungen) führt nicht notwendigerweise dazu, dass sich feministische Theorien in den Seminarplänen wiederfinden. ${ }^{7}$

»Postkolonial« sollte keinesfalls darauf reduziert werden, eine historische Epoche und einen räumlichen Bezug zu bezeichnen - dann fände der Begriff Eingang in die Friedens- und Konfliktforschung, ohne dass das herrschafts- und wissenschaftskritische Fundament postkolonialer Ansätze übernommen würde. Gerade unter

7 Die Verankerung von Gender in den Lehrangeboten und Curricula der Friedens- und Konfliktforschung im deutschsprachigen Raum gestaltet sich unterschiedlich. Während an manchen Hochschulen kaum genderbezogene Veranstaltungen angeboten werden, arbeiten andere friedenswissenschaftliche Studiengänge mit Zentren für Geschlechterforschung zusammen. In einigen Studiengängen ist die Berücksichtigung von Gender Studierenden und Lehrenden freigestellt, andere schreiben den Besuch von Lehrveranstaltungen mit »Gender-Schwerpunkt« verpflichtend vor. 
Studierenden besteht ein erhebliches Interesse an postkolonialer Theorie. Lehrangebote zu postkolonialen Ansätzen sind derzeit allerdings in den friedenswissenschaftlichen Studiengängen im deutschsprachigen Raum kaum vorhanden. Um diese Lücke zu schließen - und damit auch an aktuelle internationale Theoriedebatten anzuschließen - sind Friedens- und Konfliktforscher_innen hierzulande gefordert, sich Kenntnisse postkolonialer Perspektiven anzueignen, um diese in ihre Lehrangebote und Curricula aufnehmen zu können. Jenseits spezifischer theoretischer Ansätze gilt es, in der Lehre die Akzeptanz (erkenntnis-)theoretischer Vielfalt zu vermitteln und die Fähigkeit zur (Selbst-)Kritik als Lernziel zu etablieren - was jedoch erst dann gelingen kann, wenn die in der Lehre Tätigen sich diese Fähigkeit selbst zu Eigen machen. 


\section{Literatur}

Abrahamsen, Rita 2003: African Studies and the Postcolonial Challenge, in: African Affairs 102: 407, 189-210.

Agathangelou, Anna M./Ling, L. H. M. 2004: The House of IR: From Family Power Politics to the Poisies of Worldism, in: International Studies Review 6: 4, 21-50. Ahmad, Aijaz 1995: Postcolonialism: What's in a Name, in: Campa, Ramón de la/ Kaplan, Ann/Sprinker, Michael (Hrsg.): Late Imperial Culture, London, 11-32. Aikins, Joshua Kwesi/Bendix, Daniel 2010: Stichwort: post(-)kolonial, in: PERIPHERIE 30: 120, 478.

Alaimo, Stacy/Hekman, Susan (Hrsg.) 2007: Material Feminisms, Bloomington, IN. Amselle, Jean-Loup 1999: Ethnies et espaces: pour une anthropologie topologique, in: Amselle, Jean-Loup/M'Bokolo, Elikia (Hrsg.): Au cœur de l'ethnie. Ethnie, tribalisme et État en Afrique, Paris, 11-48.

Ashcroft, Bill/Griffiths, Gareths/Tiffin, Helen (Hrsg.) 1995: The Post-Colonial Studies Reader, London.

Ashcroft, Bill/Griffiths, Gareths/Tiffin, Helen (Hrsg.) 2000: Post-Colonial Studies: The Key Concepts, New York, NY.

Bahri, Deepika 1995: Once More with Feeling: What is Postcolonialism?, in: ARIEL: A Review of International English Literature 26: 1, 51-82.

Barkawi, Tarak/Laffey, Mark 2006: The Postcolonial Moment in Security Studies, in: Review of International Studies 32: 2, 329-352.

Batscheider, Tordis 1993: Frieden und Geschlechterverhältnis. Zur Begründung feministischer Fragestellungen in der kritischen Friedensforschung, Marburg.

Bewernitz, Torsten 2010: Konstruktionen für den Krieg? Die Darstellung von »Nation« und »Geschlecht« während des Kosovo-Konflikts 1999 in den deutschen Printmedien, Münster.

Birckenbach, Hanne-Margret 2005: Frieden, Politik und Geschlecht. Die politikund sozialwissenschaftliche Friedens- und Konfliktforschung und die Geschlechterforschung, in: Davy, Jennifer A./Hagemann, Karen/Kätzel, Ute (Hrsg.): Frieden - Gewalt - Geschlecht. Friedens- und Konfliktforschung als Geschlechterforschung, Essen, 73-95.

Blanton, Robert/Mason, T. David/Athow, Brian 2001: Colonial Style and Post-Colonial Ethnic Conflict in Africa, in: Journal of Peace Research 38: 4, 473-491.

Boehmer, Elleke/Morton, Stephen 2009: Introduction: Terror and the Postcolonial, in: Boehmer, Elleke/Morton, Stephen (Hrsg.): Terror and the Postcolonial: A Concise Companion, Malden, MA, 1-24. 
Brunner, Claudia 2010: Wissensobjekt Selbstmordattentat. Epistemische Gewalt und okzidentalistische Selbstvergewisserung in der Terrorismusforschung, Wiesbaden.

Brunner, Claudia 2012: Wissensobjekt und Sinnformel Selbstmordattentat. Über den Zusammenhang von politischer und epistemischer Gewalt am Beispiel gegenwärtiger Terrorismusforschung, in: Zeitschrift für Friedens- und Konfliktforschung 1: 1, 72-105.

Buckley-Zistel, Susanne 2007: Ethnographic Research after Violent Conflicts: Personal Reflections on Dilemmas and Challenges, in: Journal of Peace, Conflict \& Development 10/2007, 1-9.

Bussmann, Margit 2012: Quantitative Studien zu Geschlechtergleichheit und Frieden, in: Zeitschrift für Friedens- und Konfliktforschung 1: 1, 137-150.

Butler, Judith 2004: Precarious Life: The Powers of Mourning and Violence, London.

Castro Varela, María do Mar/Dhawan, Nikita 2005: Postkoloniale Theorie. Eine kritische Einführung, Bielefeld.

Castro Varela, María do Mar/Dhawan, Nikita 2009: Europa provinzialisieren? Ja, bitte! Aber wie?, in: Femina Politica 18: 2, 9-18.

Castro Varela, María do Mar/Dhawan, Nikita 2010: Mission Impossible: Postkoloniale Theorie im deutschsprachigen Raum?, in: Reuter, Julia/Villa, Paula-Irene (Hrsg.): Postkoloniale Soziologie. Empirische Befunde, theoretische Anschlüsse, politische Intervention, Bielefeld, 303-329.

Chakrabarty, Dipesh 2000: Provincialising Europe: Postcolonial Thought and Historical Difference, Princeton, NJ.

Clasen, Sarah/Zwingel, Susanne 2009: Geschlechterverhältnisse und Gewalteskalation, in: Bussmann, Margit/Hasenclever, Andreas/Schneider, Gerald (Hrsg.): Identität, Institutionen und Ökonomie. Ursachen innenpolitischer Gewalt (PVS Sonderheft 43/2009), 128-149.

Conrad, Sebastian/Randeria, Shalini (Hrsg.) 2002: Jenseits des Eurozentrismus. Postkoloniale Perspektiven in den Geschichts- und Kulturwissenschaften, Frankfurt a. M.

Creveld, Martin van 1998: Die Zukunft des Krieges, München.

Dirlik, Arif 1994: The Postcolonial Aura: Third World Criticism in the Age of Global Capitalism, in: Critical Inquiry 20: 2, 328-356.

Eriksson Baaz, Maria 1995: The Paternalism of Partnership: A Postcolonial Reading of Identity in Development Aid, London. 
Foucault, Michel 2003: »Society Must Be Defended«: Lectures at the College de France, New York, NY.

Franzki, Hannah/Aikins, Joshua Kwesi 2010: Postkoloniale Studien und kritische Sozialwissenschaft, in: Prokla 40: 1, 9-28.

Grovogui, Siba N. 2007: Postcolonialism, in: Dunne, Timothy/Kurki, Milja/Smith, Steve (Hrsg.): International Relations Theories: Discipline and Diversity, Oxford, 229-246.

Ha, Kien Nghi 2004: Ethnizität und Migration reloaded. Kulturelle Identität, Differenz und Hybridität im postkolonialen Diskurs, Berlin.

Hacker, Hanna 2012: Queer entwickeln. Feministische und postkoloniale Analysen, Wien.

Hall, Stuart 1992: The West and the Rest: Discourse and Power, in: Hall, Stuart/ Gieben, Bram (Hrsg.): Formations of Modernity, Cambridge, 275-330.

Hall, Stuart 2002: Wann gab es »das Postkoloniale«? Denken an der Grenze, in: Conrad, Sebastian/Randeria, Shalini (Hrsg.): Jenseits des Eurozentrismus. Postkoloniale Perspektiven in den Geschichts- und Kulturwissenschaften, Frankfurt a. M., 219-246.

Harders, Cilja 2005: Geschlecht und Gewaltminderung. Konfliktbearbeitung durch Veränderung von Machtverhältnissen, in: Jahn, Egbert/Fischer, Sabine/Sahm, Astrid (Hrsg.): Die Zukunft des Friedens. Band 2. Die Friedens- und Konfliktforschung aus der Perspektive der jüngeren Generationen, Wiesbaden, 495-518. Hartmann, Jürgen 2001: Internationale Beziehungen, Opladen.

Henderson, Errol A./Singer, David 2000: Civil War in the Post-Colonial World, 1946-92, in: Journal of Peace Research 37: 3, 275-299.

Kalyvas, Stathis N. 2001: »New« And »Old« Civil Wars: A Valid Distinction?, in: World Politics 54: 1, 99-118.

Kaplan, Robert 1994: The Coming Anarchy: How Scarcity, Crime, Overpopulation, and Disease are Rapidly Destroying the Social Fabric of Our Planet, in: Atlantic Monthly 273: 2, 44-76.

Kapoor, Ilan 2008: The Postcolonial Politics of Development, Milton Park.

Kerner, Ina 2007: Konstruktion und Dekonstruktion von Geschlecht: Perspektiven für einen neuen Feminismus (Gender Politik Online), in: www.fu-berlin.de/sit es/gpo/pol_theorie/Zeitgenoessische_ansaetze/KernerKonstruktion_und_Deko nstruktion/index.html; 24.02.2014. 
Kerner, Ina 2010: Verhält sich intersektional zu lokal wie postkolonial zu global? Zur Relation von postkolonialen Studien und Intersektionalitätsforschung, in: Reuter, Julia/Villa, Paula-Irene (Hrsg.): Postkoloniale Soziologie, Bielefeld, 237-258.

Klaus, Elisabeth/Kassel, Susanne 2008: Frauenrechte als Kriegslegitimation in den Medien, in: Dorer, Johanna/Geiger, Brigitte/Köpl, Regina (Hrsg.): Medien Politik - Geschichte. Feministische Befunde zur politischen Kommunikationsforschung, Wiesbaden, 266-280.

Kontzi, Kristina 2011: Postkolonialismus: Ich helfe, du hilfst,... ihnen wird geholfen, in: iz3w Nr. 323, 40-43.

Locher, Birgit 1996: Feminismus ist mehr als »political correctness «, in: Zeitschrift für Internationale Beziehungen 3: 2, 381-397.

Locher, Birgit/Prügl, Elisabeth 2001: Feminism and Constructivism: Worlds Apart or Sharing the Middle Ground?, in: International Studies Quarterly 45: 1, 111-129.

Mamdani, Mahmood 2004: Good Muslim, Bad Muslim: America, the Cold War, and the Roots of Terror, Dakar.

Marchal, Roland M. 2000: Atomisation des fins et radicalisme des moyens. De quelques conflits africains, in: Critique internationale 6/2000, 159-175.

Meyer, Jörg 2008: The Concealed Violence of Modern Peace(-Making), in: Millennium 36: 3, 555-574.

Mkandawire, Thandika 2002: The Terrible Toll of Post-colonial »Rebel Movements « in Africa: Towards an Explanation of the Violence Against the Peasantry, in: The Journal of Modern African Studies 40: 2, 181-215.

Münkler, Herfried 2002: Die neuen Kriege, Reinbek bei Hamburg.

Nachtigall, Andrea 2012: Gendering 9/11. Medien, Macht und Geschlecht im Kontext des »War on Terror «, Bielefeld.

Nachtigall, Andrea/Bewernitz, Torsten 2011: Von »Frauen und Kindern« zu »Embedded Feminism《. Frauen(rechte) als Legitimation für militärische Intervention in den Medien - Variationen einer Legitimationsfigur zwischen Kosovo-, Afghanistan- und Irakkrieg, in: Engels, Bettina/Gayer, Corinna (Hrsg.): Geschlechterverhältnisse, Frieden und Konflikt. Feministische Denkanstöße für die Friedens- und Konfliktforschung, Baden-Baden, 27-45.

Nandy, Ashis 2002: The Beautiful, Expanding Future of Poverty: Popular Economics as a Psychological Defense, in: International Studies Review 4: 2, 107-121. 
Neal, Andrew W. 2008: Goodbye War on Terror? Foucault and Butler on Discourses of Law, War and Exceptionalism, in: Dillon, Michael/Neal, Andrew W. (Hrsg.): Foucault on Politics, Security and War, Houndmills, 43-64.

Nordstrom, Carolyn (Hrsg.) 1995: Fieldwork under Fire: Contemporary Studies of Violence and Survival, Berkeley, CA.

Randeria, Shalini 1999: Geteilte Geschichte und verwobene Moderne, in: Rüsen, Jörn/Leitgeb, Hanna/Jegelka, Norbert (Hrsg.): Zukunftsentwürfe. Ideen für eine Kultur der Veränderung, Frankfurt a. M., 87-96.

Randeria, Shalini/Eckert, Andreas (Hrsg.) 2009: Vom Imperialismus zum Empire - Nicht-westliche Perspektiven auf Globalisierung, Frankfurt a. M.

Reuter, Julia/Villa, Paula-Irene (Hrsg.) 2010 a: Postkoloniale Soziologie. Empirische Befunde, theoretische Anschlüsse, politische Intervention, Bielefeld.

Reuter, Julia/Villa, Paula-Irene 2010 b: Provincializing Soziologie. Postkoloniale Theorie als Herausforderung, in: Reuter/Villa 2010 a, 1-46.

Richards, Paul 1996: Fighting for the Rain Forest: War, Youth and Resources in Sierra Leone, Oxford.

Rodríguez, Encarnación Gutiérrez 2010: Migration, Domestic Work and Affect: A Decolonial Approach on Value and the Feminization of Labor, London.

Rodríguez, Encarnación Gutiérrez/Boatca, Manuela/Costa, Sérgio (Hrsg.) 2010: Decolonizing European Sociology: Transdisciplinary Approaches, Aldershot.

Rytkonen, Helle 2006: Postcolonialism as IR Theory (Paper presented at the Annual meeting of the International Studies Association, 22.03.2006), San Diego, CA.

Smith, Linda Tuhiwai 1999: Decolonizing Methodologies: Research and Indigenous Peoples, London.

Spivak, Gayatri Chakravorty 1988: Can the Subaltern Speak?, in: Nelson, Cary/ Grossberg, Lawrence (Hrsg.): Marxism and the Interpretation of Culture, Urbana, IL, 271-313.

Steyerl, Hito/Rodríguez, Encarnación Gutiérrez 2003: Einleitung, in: Steyerl, Hito/ Rodríguez, Encarnación Gutiérrez (Hrsg.): Spricht die Subalterne deutsch? Migration und Postkoloniale Kritik, Münster, 7-6.

Sturm, Gabriele 2006: Das gesellschaftliche Geschlechterverhältnis als Konfliktfeld, in: Imbusch, Peter/Zoll, Ralf (Hrsg.): Friedens- und Konfliktforschung. Eine Einführung, Wiesbaden, 406-440.

Sylvester, Christine 1999: Development Studies and Post-colonial Studies: Disparate Tales of the Third World, in: Third World Quarterly 20: 4, 703-721. 
Wisotzki, Simone 2005: Gender und Frieden. Geschlechterperspektiven für die Friedens- und Konfliktforschung, in: Jahn, Egbert/Fischer, Sabine/Sahm, Astrid (Hrsg.): Die Zukunft des Friedens. Band 2. Die Friedens- und Konfliktforschung aus der Perspektive der jüngeren Generationen, Opladen, 111-130.

Wisotzki, Simone 2011: Geschlechterperspektiven in der Friedens- und Konfliktforschung, in: Schlotter, Peter/Wisotzki, Simone (Hrsg.): Friedens- und Konfliktforschung, Baden-Baden, 244-273.

Wood, Elisabeth Jean 2006: The Ethical Challenges of Field Research in Conflict Zones, in: Qualitative Sociology 29: 3, 373-386.

Yegenoglu, Meyda 1998: Colonial Fantasies: Towards a Feminist Reading of Orientalism, Cambridge.

Yildiz, Yasemin 1999: Keine Adresse in Deutschland?, in: Gelbin, Cathy/Konuk, Kader/Piesche, Peggy (Hrsg.): Auf-Brüche. Kulturelle Produktionen von Migrantinnen, Schwarzen und jüdischen Frauen in Deutschland, Königsstein/Taunus, 224-237.

Ziai, Aram 2011: Die Peripherie in den Sozialwissenschaften, in: Ataç, Ilker/Kraler, Albert/Ziai, Aram (Hrsg.): Politik und Peripherie. Eine politikwissenschaftliche Einführung, Wien, 24-43.

Ziai, Aram/Schaffar, Wolfram 2011: Bewaffnete Konflikte, Neue Kriege und Staatszerfall, in: Ataç, Ilker/Kraler, Albert/Ziai, Aram (Hrsg.): Politik und Peripherie. Eine politikwissenschaftliche Einführung, Wien, 172-191.

\section{Die Autorin}

Dr. Bettina Engels vertritt derzeit die Juniorprofessur Entwicklungspolitik und Politik Afrikas an der Kulturwissenschaftlichen Fakultät der Universität Bayreuth.

E-Mail: bettina.engels@fu-berlin.de 